\title{
Cognitive Radio New Dimension in Wireless Communication - State of Art
}

\author{
Rajwinder Singh \\ Dept. of Electronics Technology \\ Guru Nanak Dev University \\ Amritsar, India
}

\author{
Jasvir Singh \\ Dept. of Electronics Technology \\ Guru Nanak Dev University \\ Amritsar, India
}

\author{
A.S. Kang \\ Dept. of Electronics and Comm. \\ Panjab University \\ Chandigarh, India
}

\begin{abstract}
In today's wireless communication environment, the Radio Frequency spectrum is occupied for different purposes like cellular, Television, military, emergency or satellite communication. The frequency spectrum used for cellular communication is getting over crowded with increasing number of subscribers and demand for high data rate text or video transmission, but the frequency spectrum in other wireless broadcast and communication is not utilized efficiently, example in Television broadcast band, some of frequency spectrum is vacant at some instant or for particular time. The vacant spectrum could be used in cellular communication, means the spectrum is borrowed from Television Broadcast band to be used for cellular communication. A borrow/use of spectrum from other licensed frequency bands will improve the efficiency of spectrum use. In this paper, aspects of Cognitive Radio and comparison of different sensing techniques and methods are discussed. Secondly, the layer architecture of Cognitive Radio is presented. Thirdly, hardware and software platforms are discussed for Cognitive Radio testing.
\end{abstract}

\section{Keywords}

Cognitive Radio, Software Defined Radio, Spectrum Sensing, Primary User, Secondary User, Transmit Power Control and Dynamic Spectrum Management.

\section{INTRODUCTION}

The Cognitive Radio is a radio that can borrow/use spectrum by sensing vacant or white space in spectrum from their surroundings, adapt it and can change frequency dynamically based on its location and other neighbouring radios by learning their spectrum pattern. Ability of CR is to recognize spectrum availability and reconfigure itself for efficient spectrum use. CR provides more radio spectrum for cellular communication, more bandwidth as per requirement for transmission, and interoperability between communication systems.

By the mean of Cognitive Radio technology, the high data rate could be achieved for multimedia applications. Cognitive Radio covers the services such as speaker recognition, language identification then translation into another, text-tospeech conversion, speech-to-text conversion, noise suppression, and noise management. For military applications, Cognitive Radio will allow finding free channel automatically to communicate instead of manually setting to a particular radio channel. Another benefit, CR can resist the jamming in a particular area where radio signal jammers are used and some radio spectrum is restricted. In disaster and emergency condition, due to over-crowding of the radio spectrum collapses the communication system. In such circumstances when radio spectrum is completely occupied and no direct link is available to the access point. But through other CR terminals, access point is reachable by forming spontaneous network. Cognitive Radio is discussed in the following sessions: in session 2-history of Cognitive Radio is discussed in flow of technologies from analog domain to intelligent domain, in session 3-working cycle of CR and its sensing methods and techniques are discussed, in session 4architecture of $\mathrm{CR}$ is discussed in accordance with its functions layer by layer, in session 5-hardware platforms and testbeds which are established for testing of Cognitive Radio, and in session6-software platforms are discussed for implementation of Cognitive Radio.

\section{HISTORY OF COGNITIVE RADIO}

The roadmap to Cognitive Radio technology starts from analog domain to intelligent domain in the following phases as shown in figure 1 .

a) Analog Radio

Analog is the standard method of radio broadcasting where the entire spectrum of radio frequencies is transmitted in a continuous flow of waveform data with amplitude or frequency modulation technique. Nikola Tesla wirelessly transmitted electromagnetic energy. Guglielmo Marconi sent first wireless signal across Englishchannel in 1899 and Jagadish Chandra Bose's 1904 US patent described Galena Crystal detector for demodulating continuous wave radio signals [1].

b) Digital Radio

Digital radio describes radio communications technologies which carry information as a digital signal, by means of digital modulation techniques. The baseband signal implemented on a Digital Signal Processing.

c) Software Capable Radio

The radio is capable of fixed modulation and limited data rate but it is software controlled means data handling is done through software. It has the capability to select channel automatically and programmable cryptography of data.

d) Software Programmable Radio

In this technology, the new features and functions can be added by changing in software module. It has networking capability and multi waveform interoperability [2].

e) Software Defined Radio

The complete processing in SDR technology is through software in which functionalities are performed by software modules running on field programmable gate arrays (FPGAs), digital signal processors (DSP), general-purpose processors 
(GPP), or a combination of them. By software updating or changing it, we can change its operating parameters and characteristics such as coding technique, modulation type. The majority of components are software implemented instead of hardware implemented [3]. We can re-configure the radio to new functions and operations manually but it can be done on demand only. It can't re-configure itself without its user even knowing it.

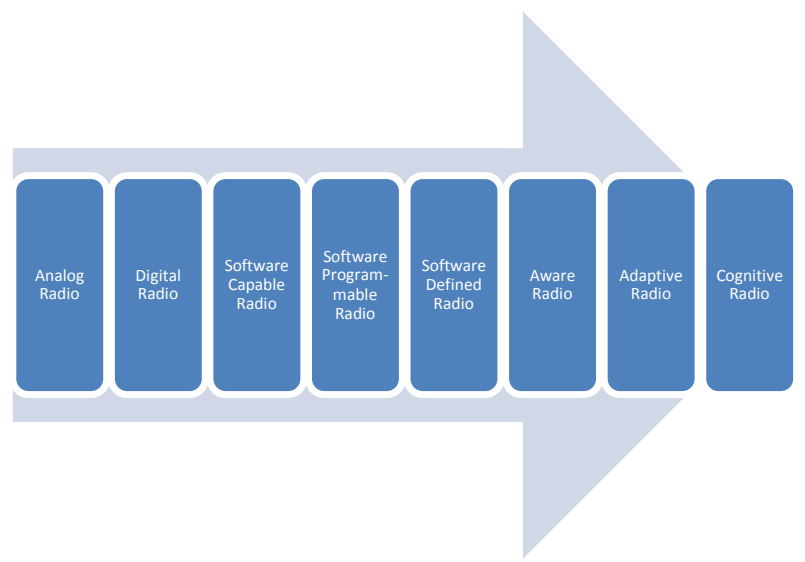

Fig 1: Roadmap of CR from Analog domain to Intelligent domain

f) Aware Radio

Aware radio is a radio which uses sensors to gather the information about neighbouring wireless radio environment to estimate the interference temperature, Quality of Service and other service parameters [2]

g) Adaptive Radio

Adaptive Radio is a radio that can adjust and modify their operating parameters such as frequency, bandwidth, modulation scheme, error correction coding, transmit power, and filtering characteristics; to improve its performance by using sensor inputs to find appropriate parameters [2].

h) Driver of Cognitive Radio

Joseph Mitola III outlined a cognition process through which radio can reconfigure itself through the phases of process as awareness, perception, reasoning, and decision making $[4,5]$. Figure 2 shows the CR terminal-evolution. The figure presents three main parts: the Software Radio (SR), the Software Defined Radio (SDR), and the CR terminals, underlying thus the $\mathrm{CR}$ predecessors and improvements as per Mitola's view.

\begin{tabular}{|c|c|c|}
\hline \multirow[b]{2}{*}{$\begin{array}{c}\text { Software } \\
\text { Radio }\end{array}$} & \multirow{3}{*}{$\begin{array}{r}\text { Software } \\
\text { implementation } \\
\text { instead of hardware } \\
\text { implementation for } \\
\text { jority of components }\end{array}$} & \multirow{3}{*}{$\begin{array}{l}\text { Knowledge } \\
\text { Reasoning } \\
\text { Decision }\end{array}$} \\
\hline & & \\
\hline & & \\
\hline \multicolumn{2}{|c|}{ Software Defined Radio } & \\
\hline \multicolumn{3}{|c|}{ Cognitive Radio } \\
\hline
\end{tabular}

Fig 2: The CR terminal-evolution [6]

\section{WORKING CYCLE OF CR}

In general the cognitive cycle is a continuous process comprising of the following phases a) Sensing, b) Analysis, c) Reasoning and d) Adaptation, as described in Figure 3. The dominant phase of process of the cognitive cycle is sensing the radio spectrum and other phases focus on how to use the radio spectrum based on its ability.

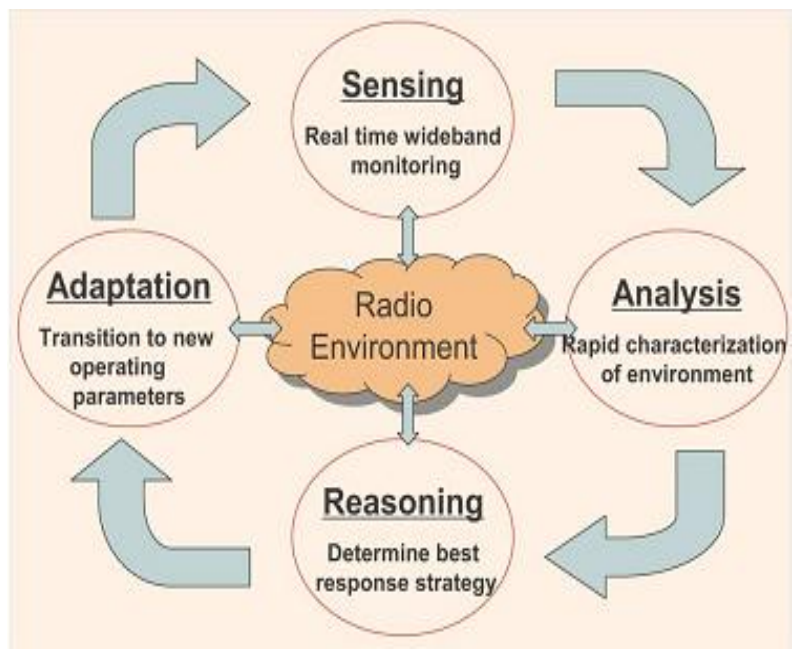

Fig 3: Cognitive Cycle [8]

a) Sensing-the ability to observe and estimate the state of the radio environment such as spectrum vacancy or white space. Sensing is necessary phase in cognitive radio to change its operation based on its current knowledge of RF environment. b) Analysis- characterization of sensed spectrum and recognize patterns.

c) Reasoning- determines the best use of sensed spectrum in operating parameters such as modulation technique, power control, etc and to meet the requirements of the user.

d) Adaptation- radio changes its operation behavior in accordance with the resulted analysis of a radio environment and the change is not only based on pre-coded algorithms but also as a result of a learning mechanism.

The major tasks of Cognitive Radio to work are divided into transmission and reception side but as part of single device. Firstly, the spectrum sensing and analysis at the receiver detects the vacant or white spectrum and predicts modeling of environment around the receiver. Secondly, the channel identification on the receiver side is required for coherent detection of the message signal. Thirdly, transit power control at the transmitter side is to manage the power in underlay or overlay approach by using information from spectrum sensing and analysis. Finally, dynamic spectrum management on the transmitter side makes decision on the use of spectrum so that the interference temperature at the primary users should not exceed the prescribed limit. In figure 4 , the tasks of cognitive radio are shown on both transmission and reception side as single radio.

\subsection{Spectrum Sensing and Analysis}

By spectrum sensing, Cognitive Radio has information about its surrounding environment from which it can know about presence of primary user and which part of spectrum is used by primary user or left vacant. Spectrum sensing and analysis encompasses spectrum awareness which is classified into two parts: passive and active awareness. 


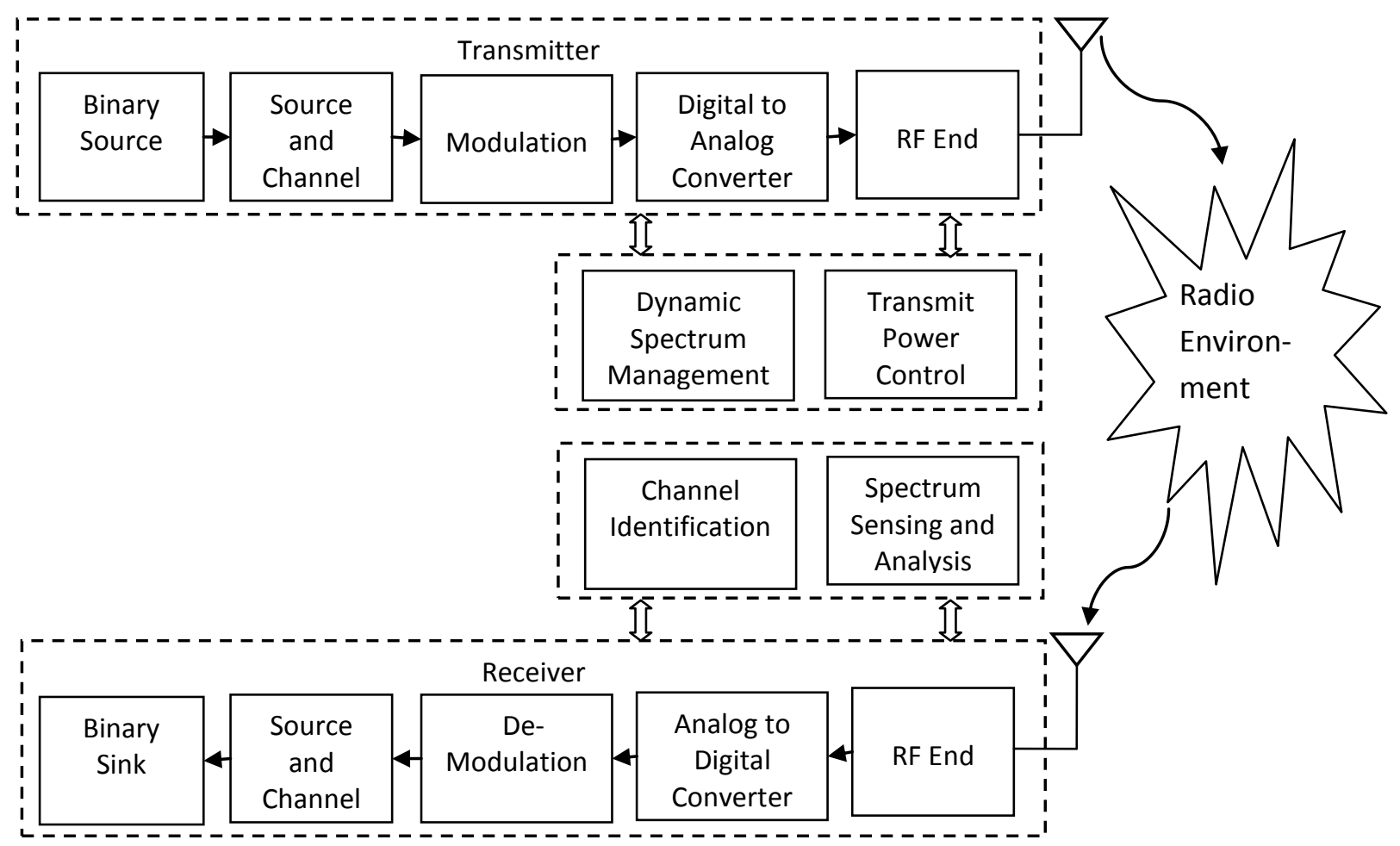

Fig 4: Tasks of cognitive radio

\subsubsection{Passive Awareness}

Cognitive Radio has knowledge about radio environment of primary communication system without sensing their spectrum pattern. The spectrum use pattern of a primary communication system is shared among the secondary communication systems or secondary user within same communication system. The passive awareness is classified as negotiated, policy based, database and server approach [9]. Advantage of passive approach, it provides the interference free communication, information about spectrum is shared by either radio regulatory or particular communication system and disadvantage, channels required to broadcast and to access the information about spectrum and management of information is required such as to mark the spectrum is utilised or not by secondary user in a real time.

\subsubsection{Active Awareness}

The spectrum is actively sensed by secondary users from its surrounding wireless radio environment and based on that spectrum information, use of spectrum is decided. The active awareness is more real time approach requires constant monitoring of the channel so that new primary users and possible vacant channels will be detected. Active Awareness is classified into three types; first is non-cooperative sensing, second is cooperative Sensing and last one is interference based sensing.

\subsubsection{Non-Cooperative Sensing}

Each secondary user makes its decision individually from the information gathered by sensing about the radio spectrum environment. It is also known as local sensing. There is hidden node problem due to obstacle between the primary and secondary users [9].

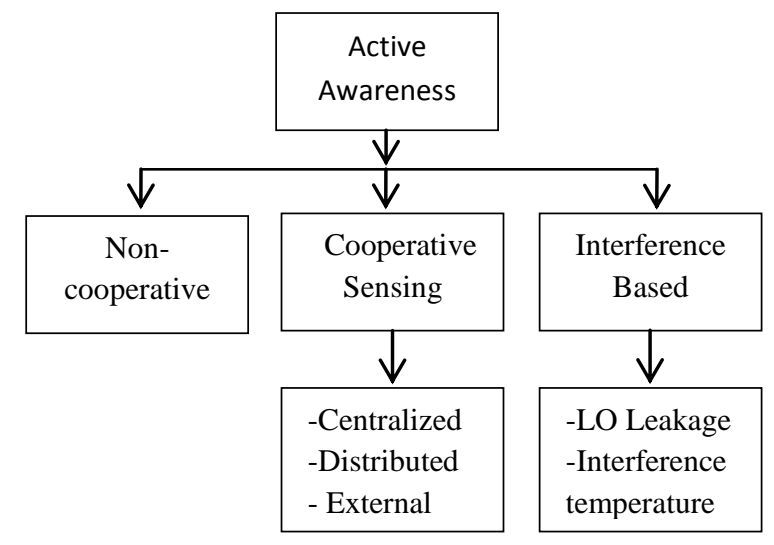

Fig 5: Classification of Active Awareness

\subsubsection{Cooperative Sensing}

Spectrum information gathered by different secondary users is shared among them and decision of a vacant spectrum is made by correlating the information received about primary user spectrum. Cooperative sensing curtails the chances of misdetection and false alarm. Cooperation can solve hidden node problem and it also decrease sensing time [9].

Classification of cooperative sensing:-

(a) Centralized Sensing

The sensing information from all secondary users is gathered at one server, and it will broadcast the information to other secondary users in its vicinity.

(b) Distributed Sensing

The secondary users share information among each other and will make their own decisions about occupation of radio 
spectrum. Distributed sensing will reduce the cost of the communication system because there is no need of central unit or server.

(c) External Sensing

An external device with no urge to use the spectrum, performs the sensing and broadcasts the spectrum occupancy information to cognitive radios. The main advantages of external sensing are overcoming hidden node problem and the uncertainty due to non-time dispersive fading.

Table 1: Comparison of non-cooperative and cooperative sensing methods [10]

\begin{tabular}{|c|l|l|}
\hline Sensing method & \multicolumn{1}{|c|}{ Advantage } & \multicolumn{1}{c|}{ Disadvantage } \\
\hline $\begin{array}{c}\text { Non-cooperative } \\
\text { sensing }\end{array}$ & $\begin{array}{l}\text {-Computational and } \\
\text { implementation } \\
\text { simplicity }\end{array}$ & $\begin{array}{l}\text {-Hidden node } \\
\text { problem } \\
\text {-Multipath and } \\
\text { shadowing }\end{array}$ \\
\hline Cooperative \\
sensing & $\begin{array}{l}\text {-Higher accuracy } \\
\text {-Reduced sensing } \\
\text { time }\end{array}$ & $\begin{array}{l}\text {-Complex } \\
\text {-Traffic overhead } \\
\text {-Shadoed for a control } \\
\text { channel }\end{array}$ \\
& $\begin{array}{l}\text { and hidden } \\
\text { node problems can } \\
\text { be prevented }\end{array}$ & \\
\hline
\end{tabular}

\subsubsection{Interference Based Sensing}

In Interference Based Sensing, the interference around the secondary cognitive radio is measured to find out the vacant radio spectrum for communication. It is receiver centric sensing of the radio spectrum. This sensing is mostly useful in spectrum underlay approach for UBW. Interference based sensing is categorized into two types; one is LO leakage based approach and other is interference temperature based approach.

(a) LO Leakage Based Approach

In this approach, primary receiver is detected from its emission of the local oscillator (LO) leakage power from its $\mathrm{RF}$ front end while receiving the data from primary transmitter. Disadvantage, it can be only used in short range distance to primary receiver and took long detection time to achieve accuracy [11]. This approach can be used for spectrum overlay power control technique.

(b) Interference Temperature Based Approach

The interference temperature is calculated from the interfering surroundings of the secondary user and compared with mandatory interference threshold that can be tolerated referred by radio regulatory. If the interference temperature is less than that threshold or maintaining it low, then secondary user can use the spectrum of or co-exist with the primary user [9]

The most common sensing techniques used for active awareness either for cooperative or non-cooperative sensing in Cognitive Radios are briefly explained in table below:

Table 2: Comparison of sensing techniques used in cooperative and non-cooperative based sensing.

\begin{tabular}{|l|l|l|l|}
\hline & \multicolumn{1}{|c|}{ Techniques } & \multicolumn{1}{|c|}{ Advantages } & \multicolumn{1}{|c|}{ Disadvantages } \\
\hline 1. & Energy & -Low & -Inability to differ- \\
Detection & computational & entiate interference \\
Based & and & or noise from \\
Approach & implementation & primary signal \\
-detected signal & complexities & -Can't distinguish \\
energy & -No need of & between different \\
compared with & knowledge & types of \\
a threshold & about primary & transmissions \\
\hline
\end{tabular}

\begin{tabular}{|c|c|c|c|}
\hline & & $\begin{array}{l}\text { user } \\
\text { transmission }\end{array}$ & $\begin{array}{l}\text {-Low performance } \\
\text { at lower SNR } \\
\text {-Not effective in } \\
\text { spread spectrum } \\
\text { detection }\end{array}$ \\
\hline 2. & $\begin{array}{l}\text { Cyclostationary } \\
\text { Based } \\
\text { Approach } \\
\text {-autocorrelation } \\
\text { of received } \\
\text { signal which is } \\
\text { periodic } \\
\text { function of time }\end{array}$ & $\begin{array}{l}- \text {-Differentiate } \\
\text { noise from } \\
\text { primary signal } \\
\text {-Distinguish } \\
\text { different types } \\
\text { of transmissions } \\
\text { and primary } \\
\text { users at } \\
\text {-Robust at } \\
\text { lower SNR }\end{array}$ & $\begin{array}{l}\text {-Require partial } \\
\text { knowledge of } \\
\text { primary signal } \\
\text {-High } \\
\text { computational cost } \\
\text {-Long observation } \\
\text { time }\end{array}$ \\
\hline 3. & $\begin{array}{l}\text { Waveform } \\
\text { Based } \\
\text { Approach } \\
\text {-correlate the } \\
\text { received signal } \\
\text { with a known } \\
\text { signal } \\
\end{array}$ & $\begin{array}{l}\text {-More reliable } \\
\text {-Performance } \\
\text { increases with } \\
\text { length of known } \\
\text { signal pattern } \\
\text { increases }\end{array}$ & $\begin{array}{l}\text {-Signal patterns } \\
\text { such as preamble, } \\
\text { pilot positioning, } \\
\text { spread sequence } \\
\text { should known }\end{array}$ \\
\hline 4. & $\begin{array}{l}\text { Radio } \\
\text { Identification } \\
\text { Based } \\
\text { Approach } \\
\text {-identify the } \\
\text { transmission } \\
\text { technology by } \\
\text { used primary users } \\
\text { prim }\end{array}$ & $\begin{array}{l}\text {-Higher } \\
\text { accuracy and } \\
\text { higher } \\
\text { dimensional } \\
\text { knowledge } \\
\text { about signal }\end{array}$ & -More complex \\
\hline 5. & $\begin{array}{l}\text { Matched Filter } \\
\text { Based } \\
\text { Approach } \\
\text {-correlate a } \\
\text { known signal } \\
\text { with an } \\
\text { unknown signal } \\
\text { to detect the } \\
\text { presence }\end{array}$ & $\begin{array}{l}\text {-Short time to } \\
\text { achieve } \\
\text { probability of } \\
\text { misdetection } \\
\text {-Maximize } \\
\text { SNR }\end{array}$ & $\begin{array}{l}\text {-Require priori } \\
\text { knowledge about } \\
\text { signal features } \\
\text { such as bandwidth, } \\
\text { frequency, } \\
\text { modulation and } \\
\text { frame format } \\
\text {-Require } \\
\text { demodulate } \\
\text { received signal for } \\
\text { which channel } \\
\text { synchronization is } \\
\text { required } \\
\text {-More complex } \\
\text { with increase in } \\
\text { type of primary } \\
\text { signals power } \\
\text {-High } \\
\text { consumption[12] }\end{array}$ \\
\hline 6. & $\begin{array}{l}\text { Multi Taper } \\
\text { Based } \\
\text { Approach }\end{array}$ & $\begin{array}{l}\text {-employs a set } \\
\text { of orthogonal } \\
\text { data tapers to } \\
\text { mitigate the loss } \\
\text { of } \\
\text { information[16] }\end{array}$ & 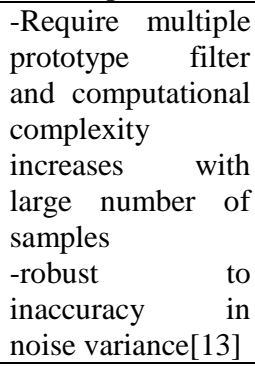 \\
\hline 7. & $\begin{array}{l}\text { Wavelet Based } \\
\text { Approach } \\
\text {-detect edges in } \\
\text { the Power } \\
\text { Spectral } \\
\text { Density (PSD) }\end{array}$ & $\begin{array}{l}\text {-Effective for } \\
\text { wide band } \\
\text { signals } \\
\text {-more flexible } \\
\text {-reduce } \\
\text { computational } \\
\text { complexity }\end{array}$ & $\begin{array}{l}\text {-Not work for } \\
\text { spread } \\
\text { spectrums[14] }\end{array}$ \\
\hline
\end{tabular}


The energy detection based approach is least accurate and complex; and the most accurate and complex approach is matched filter approach. The waveform based approach is as much accurate as matched filter approach but less complexity than it. The matched filter approach requires a high degree of prior knowledge to achieve higher accuracy.

\subsection{Channel Identification}

Channel identification is a part of the receiver side which is essential for coherent reception. Channel identification is helpful for the computation of the radio channel capacity and for the power control algorithms at the transmitter side. By channel identification algorithms for adaptive estimation of the state of a fast fading channel are also estimated in CR communication system [7].

\subsection{Dynamic Spectrum Management And Transmit-Power Control}

Cognitive Radio needs to select or manage the transmission parameters such as vacant radio channels and power levels, in that way the transmission of secondary user will not hinder the primary user and coexist with it within the interference temperature level [15]. Power control, bit rate control, and dynamic spectrum management are the tasks which are performed on the transmitter side [7, 16]. Transmit power control is a feature that enables a device to dynamically switch between several transmission power levels according to environment status such as path loss, interference value, SNR thresholds, link data rate etc. It allows transmission at the allowable limits when necessary, but reduces the transmitter power to a lower level to allow greater sharing of spectrum when higher power operation is not necessary [17, 18]. The transmissions techniques can be classified into two types: overlay and underlay approach.

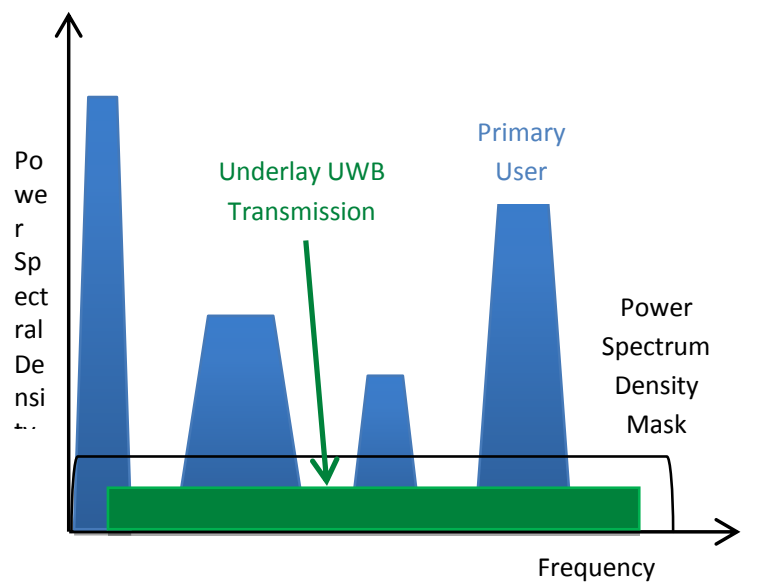

Fig 6: Underlay Approach [19]

The underlay approach allows primary and secondary users transmit simultaneously and secondary user transmits at lower power by enforcing a spectral mask so that the interference temperature by the secondary users is below the acceptable level of interference to primary users. The secondary user can use the radio spectrum as a spread over the whole spectrum which is confined to short-range communications such as ultra wideband (UWB) systems.

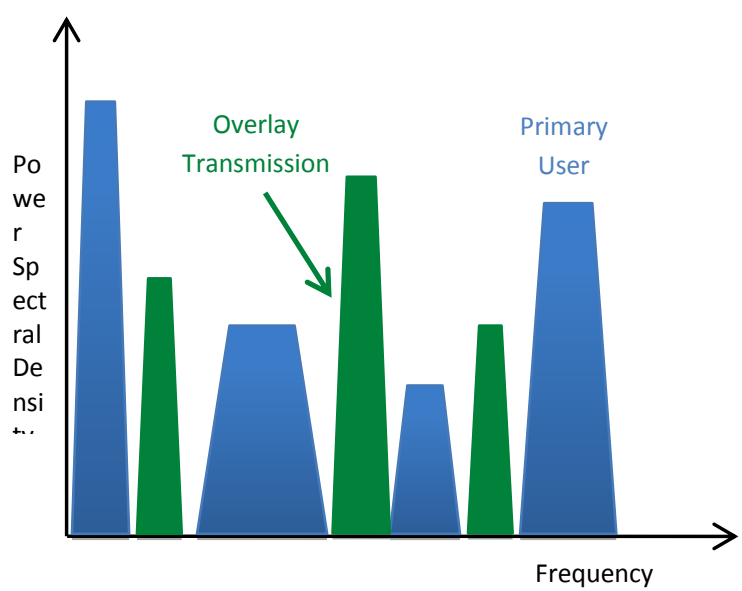

Fig 7: Overlay Approach [19]

In overlay approach, secondary users transmit with different power levels within spectrum holes or white space simultaneously and in accordance with the power level of primary users within limits of signal-to-noise ratio. By appropriate splitting of power, the increase of the primary user's SNR can offset the decrease of the primary user's SNR due to the interference generated by the secondary users.

\section{ARCHITECTURE OF CR}

Cognitive Radio architecture is a framework which defines different functions of the cognitive radio in accordance with layers reference model and this will facilitate to establish radio product with standard design so that different products from different manufacturers can communicate with each other [20]. The architecture of Cognitive Radio is represented in figure 8 as per its functions layer by layer of Open System Interconnection stack.

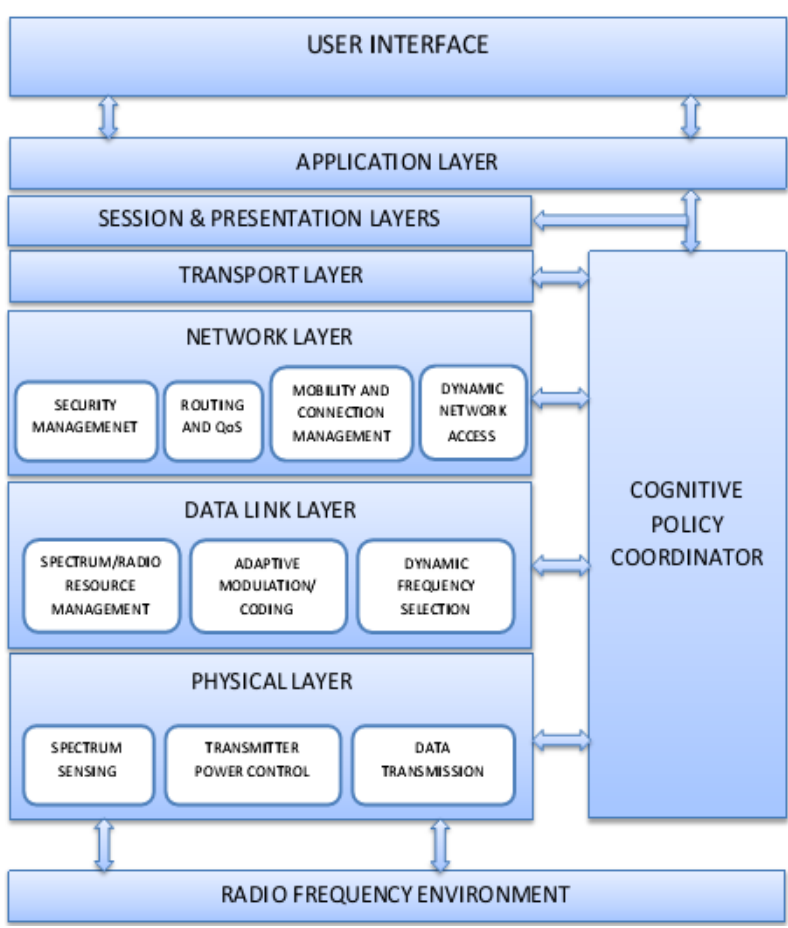

Fig 8: Cognitive Radio Architecture 
The physical layer consists of three main functions, first is spectrum sensing that is detecting white space, second is transmitter power control like in underlay and beam-forming, and third is data transmission (data rate, fading statistics).

The data link layer should perform three functions as spectrum/radio resource management, adaptive modulation or coding and dynamic frequency selection. Spectrum/Radio Resource Management is a part of second layer to efficiently manage and organize spectrum vacancy information among cognitive radios, effective spectrum management schemes are necessary in a communication system for high QoS requirements. Dynamic Frequency Selection is defined as a mechanism that dynamically detects signals from other radio frequency communication systems and selects such a frequency and polarization to avoid co-channel operation with other systems. A cognitive radio could select the appropriate modulation and coding type for use within a particular transmission system to permit interoperability between different communication systems and to adapt to the previous determined data composition.

The network layer performs four functions as security management, routing \& Quality of Service, spectrum mobility and connection management, and dynamic network access. Since Cognitive radios will work with different radio spectrum, so cognitive radio network is a heterogeneous network in nature, various heterogeneities such as wireless access technologies introduce lots of security issues. Security management is needed in the radio to provide trust worthy communication. Due to the heterogeneity of Cognitive Radio Networks, routing and topology information at network nodes will get complex [17-18]. Good mobility and connection management can help neighborhood discovery and detect available service, which help cognitive radios to select route and networks. Spectrum mobility is a process in which Cognitive Radios change its frequency of operation that must be seamless.

The radio domain information consists of Radio Frequency (RF) and environmental data that could affect system performance such as propagation or interference sources. The cognitive policy coordinator provides policy-related information which helps the cognitive radio decide on allowable/legal solutions and blocks any solutions that break local regulations.

\section{CR HARWARE PLATFORMS AND TESTBEDS}

For research, testbeds have been made to test point-to-point communication link and measurements are made such as transmission data rate, bit error rate, Signal to Noise ratio and spectrum detection under different propagation environments. There are some Cognitive Radio testbeds as following:

\subsection{Berkeley Emulation Engine}

The BWRC (Berkeley Wireless Research Centre) cognitive radio hardware test bed consists of a Berkeley Emulation Engine (BEE2), reconfigurable $2.4 \mathrm{GHz}$ radio modems, and fibre link interface for connection between BEE2 and radios [21]. Real-time baseband processing implemented using BEE2 consists of five Xilinx Vertex-2 Pro 70 field programmable gate arrays (FPGA). These five FPGAs provide parallel computation and offer rapid re-configurability [22].

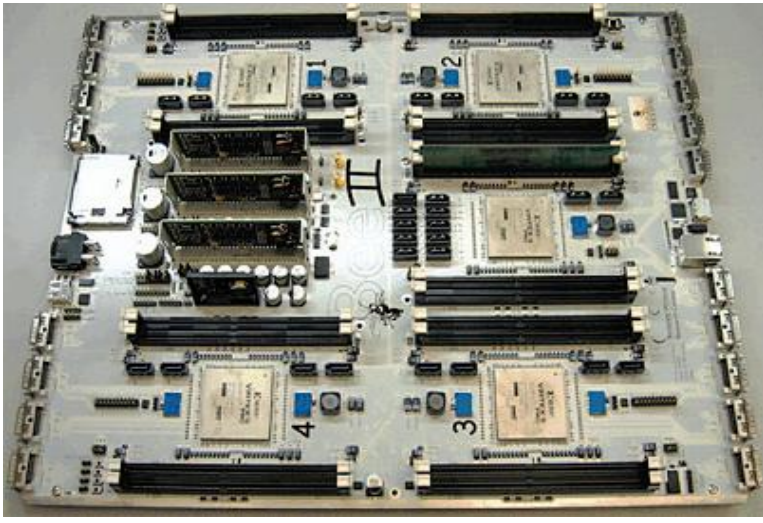

Fig 9: BEE2 Processing Board

\subsection{Motorola 10 MHZ-4 GHz CMOS-Based Experimental CR Platform}

The Radio Frequency of integrated circuit has programmed to receive and transmit signals of wireless protocols at carrier frequencies from $10 \mathrm{MHz}$ to $4 \mathrm{GHz}$ with channel bandwidths from $8 \mathrm{kHz}$ to $20 \mathrm{MHz}$. Advantage, it can perform cyclostationary analysis, a type of spectrum sensing used in cognitive radio to assess on-air activity. Disadvantage, it can lead to spotty coverage that targets a subset of predefined frequencies [23-24].

\subsection{Maynooth Adaptable Radio System}

The MARS platform is a personal computer-connected radio front end and signal processing is implemented on the computer's processor. The platform can simulate wireless communication standards in the frequency range 1700 to 2450 MHz. This platform was developed to support a number of wireless communication modes such as IEEE $802.11 \mathrm{~b}$, GSM1800, GSM1900, and UMTS/CDMA.

\subsection{CalRadio 1}

The CalRadio 1 platform is a standalone board that can potentially provide the functionalities for fast development and testing of communication algorithms and protocols. CalRadio 1 is equipped with a 802.11b PHY layer in hardware and the MAC layer is run on DSP which can be reprogrammed in standard ANSI C [25]. The CalRadio 1 platform consists of four main components, an ARM (ARMv7TDMI) processor, a Digital Signal Processor (TI 5471 DSP) [26], a Baseband (BB) processor (Intersil HFA3863) and an RF transceiver (MAX28281).

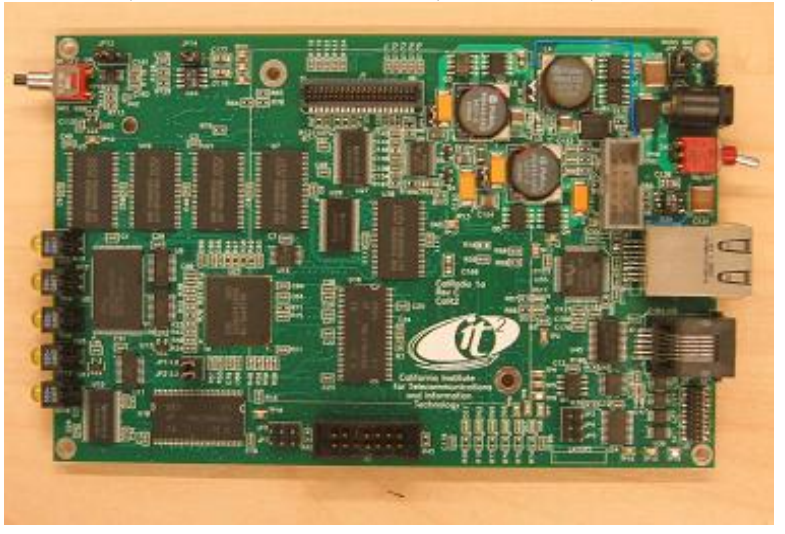

Fig 10: CalRadio1 mainboard [27] 
Both processor, ARM and DSP are integrated on a single dual-core chip (TMS320VC5471) produced by Texas Instruments. As comparing with Open System Interconnection stack, the ARM processor performs the higher layer functionalities; and the DSP carries out the data link layer (DLL) functionalities, and the physical layer (PHY) functionalities are implemented by the Baseband processor and the RF transceiver [27].

\subsection{Kansas University Agile Radio (KUAR) Platform}

The KUAR is a software-defined radio platform which operates in the $5-6 \mathrm{GHz}$ band and is capable of implementing numerous modulation algorithms, media access control (MAC) protocols, adaptation mechanisms and interface wired networks. The KUAR consists of five major sub-systems on three printed circuit boards: a power supply, a control processor (Intel Pentium-M: $1.4 \mathrm{GHz}$ ), a digital board with a programmable signal processor (Xilinx Vertex II Pro V30), A/D (LTC2284), and D/A (AD9777) converters, an RF transceiver, and antennas. It is compatible with GNU radio software [28-29].

\subsection{Lyrtech SFF SDR Development Platform}

The Small Form Factor (SFF) Software-Defined Radio (SDR) Development Platform is a portable SDR platform to address the need of the military and commercial mobile communication. It is designed of the DSP (DM6446) and FPGA (Virtex-4 SX35) technology.

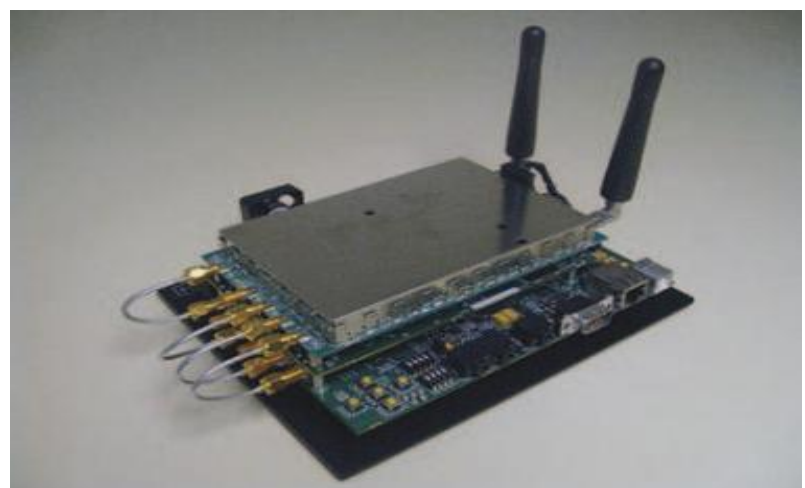

Fig 11: SFF SDR DP with low-band Tunable RF module [30]

A high-level software development tools are offered with the SFF SDR: a model-based design kit, which gives access to Lyrtech development board interfaces from within the MATLAB/Simulink model-based design environment, and an SCA core framework. The femto BTS prototype can be simulated using the platform. Its computing capacity is fixed and it's up gradation is not easy to meet the needs of Cognitive Radio Network Test-beds. The minimum response delay of SFF SDR DP is about tens of milliseconds and remains constant through-out [31].

\subsection{Universal Software Radio Peripheral (USRP)}

Universal software radio peripheral (USRP) and USRP2 by Ettus Research are widely used hardware platforms in the area of Cognitive Radio. In the USRP, high sample-rate processing takes place in the Xilinx Spartan-3 XC3S2000 field programmable gate array (FPGA), while lower sample-rate processing happens in the host computer. The two on-board digital down-converters (DDCs) mix, filter, and decimate (from $64 \mathrm{MS} / \mathrm{s}$ ) incoming signals in the FPGA. The DDCs and DUCs, both also simplify analog filtering requirements. The USRP is frequently used with GNU Radio software but some researchers have integrated the USRP into the LabView and Matlab/Simulink environments [32].

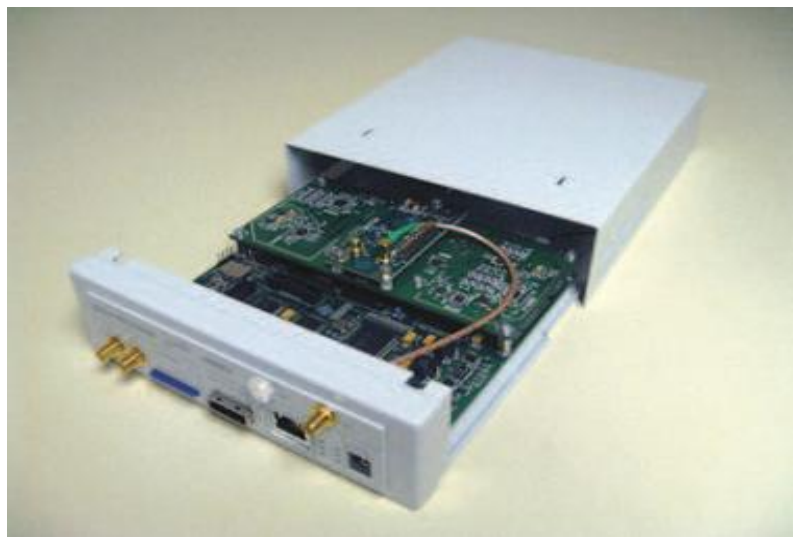

Fig 12:USRP2 with WBX RF daughterboard [30]

\subsection{WARP: Wireless Open-Access Research Platform}

The platform is capable of implementing advanced wireless algorithms. The platform consists of both custom hardware and FPGA implementations of key communication blocks. Optimized and documented designs are implementing various standard functional units. These include both generic blocks, like high speed correlators and multi-rate filters, as well as application specific blocks like OFDM packet detection and symbol synchronization. Current designs include implementations of full network layers, like an OFDM PHY and a simple MAC protocol [33].

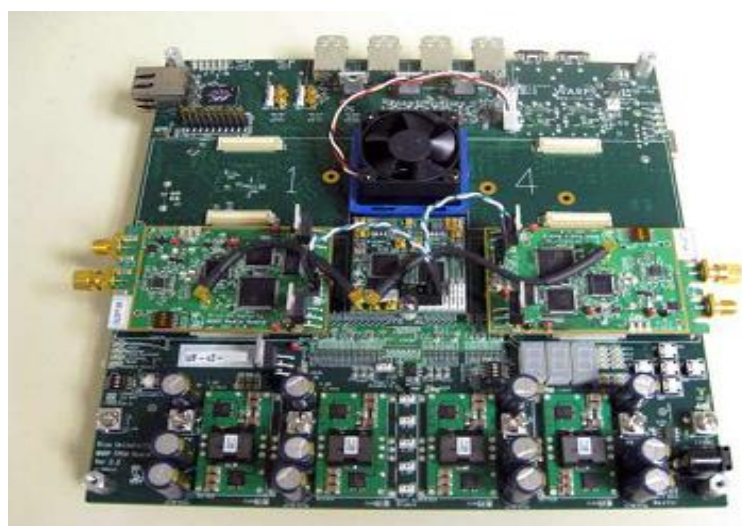

Fig 13: WARP FPGA board with two radio boards [30]

We can interact with WARP nodes directly from the MATLAB workspace and samples created in MATLAB are stored in the FPGA and that samples in the FPGA are sent over-the-air using the radios. The only disadvantage of WARP is that Virtex-4 FPGA used is not powerful enough to accommodate both transmitter and receiver functions simultaneously, therefore full-duplex communications as desired by Cognitive Radio Network testbeds cannot be implemented just one WAPR. 


\subsection{Microsoft Research Software Radio}

The Software Radio (Sora) platform was developed by Microsoft. Sora consists of two boards Radio Control Board (RCB) and RF board; it is a computer-aided platform. The RCB contains a Xilinx Virtex-5 FPGA, and it interfaces with a host computer through a peripheral component interconnect express (PCIE) interface. RCB board is an interface board for transferring digital signals between the RF board and host computer. Advantage, it fulfills a high-throughput interface between an RF board and a host computer [34]. The processing work is conducted on the host computer; it has to be enough powerful for real time computations. Implementation of full duplex communication is also challenging.

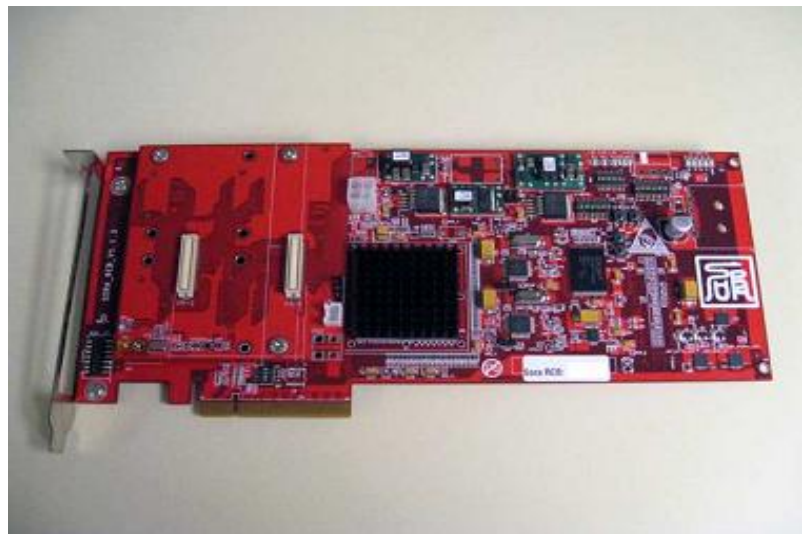

Fig 14: Sora radio control board [30]

\section{SOFTWARES COMPATIBLE WITH TESTBEDS AND ALONE VIRTUAL SIMULATORS}

\subsection{SystemVue}

SystemVue is a system level design environment which enables system architects and algorithm developers to innovate physical layer of wireless with number of libraries such as DSP, communications (W1461), RF (W1464), adaptive (W1904), FPGA (W1462) and others. The adaptive library provides SystemVue tokens for virtually the full spectrum of adaptive signal processing algorithms ranging from simple least mean squares (LMS), to recursive least squares (RLS), to Kalman filters. The library has been designed to allow the most common tokens to be easily integrated into a SystemVue design simulation.

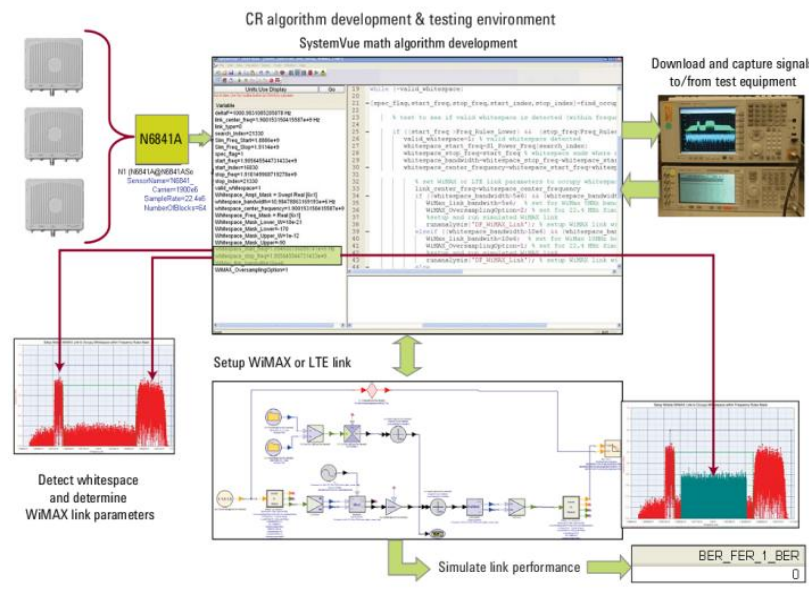

Fig 15: SystemVue's developed and tested CR whitespace algorithm [35]
The software adapt library provides convenient, flexible and easy to use SystemVue tokens to implement well known adaptive algorithms for the many DSP and communications systems applications that currently exist. A communication library includes LTE Advanced, WiMAX, OFDM, Zigbee, $3 \mathrm{G}$ such as GSM, CDMA and other blocksets. In figure 15, from live radio environment spectrum is sensed using N6841A sensors and whitespace is used for either WiMAX or LTE link [35].

\subsection{GNU Radio}

GNU Radio Companion (GRC) is a graphical user interface was developed by Eric Blossom. In GNU Radio for analysis and wave formations, various pre-assembled signal processing blocks exist. GNU radio is capable of generating and receiving various kinds of waveforms such as NBFM, WBFM, PSK, etc. It is compatible with the USRP2 hardware and operating systems like MAC, Linux and OS X for Cognitive Radio design. In GNU radio, signal processing code is written in $\mathrm{C}++$; Control and GUI code is written in python. FPGA code is written in Verilog and this can also combined with VHDL [36].

\subsection{MATLAB}

It is a high-level technical computing language and interactive environment for data analysis and visualization, algorithm development and numerical computation. Wide range of applications of MATLAB like signal and image processing, communications, design control, testing and measurements, modeling and analysis broaden its working environment. Even more add-on toolboxes extend the MATLAB environment to solve particular classes of problems in these application areas. Integration of MATLAB code with other languages and applications is also an amazing feature. MATLAB Simulink provides an environment for multi domain simulation and Model-Based Design for dynamic and embedded systems. Interactive graphical environment with customizable set of block libraries provides an environment to design, simulate, implement, and experiment as virtual laboratory for communications, controls, signal processing which is quiet important for Cognitive Radio implementation. Moreover integration of Simulink with MATLAB, providing immediate access to an extensive range of tools to develop algorithms, analyses and visualizes simulations. Moreover we can customize the modeling environment by defining signal and customizing its parameters and further testing the data.

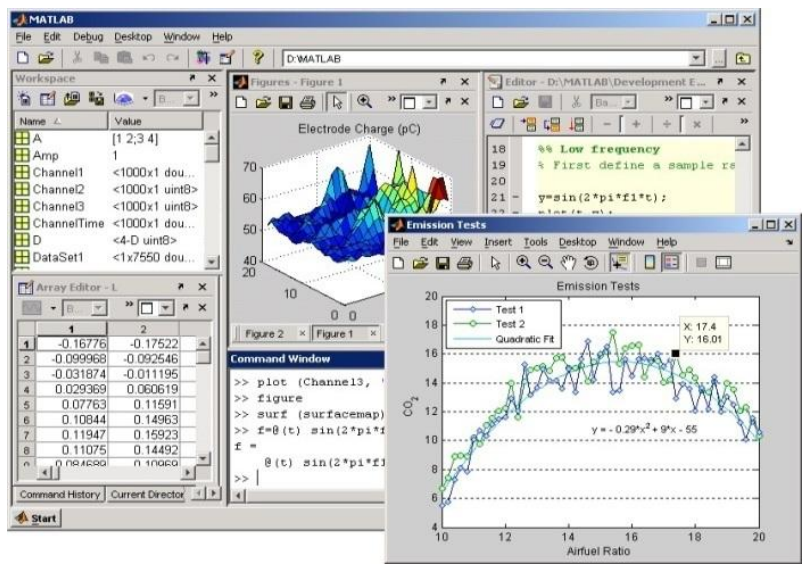

Fig 16: Matlab workspace, command window, editor and graphical output. 


\subsection{SEAMCAT}

SEAMCAT (Spectrum Engineering Advanced Monte Carlo Analysis Tool) is a statistical simulation model that is implementation of Monte Carlo analysis method to address the potential interference between different or within same radio communication systems. The communication system with cognitive radio can be implemented within different wireless radio propagation models. The sharing of channels by secondary users with energy detection spectrum sensing technique, power control and interference limits can be evaluated. It is an open source for spectrum engineering and radio compatibility problems; and compatible with both Windows and Linux [37].

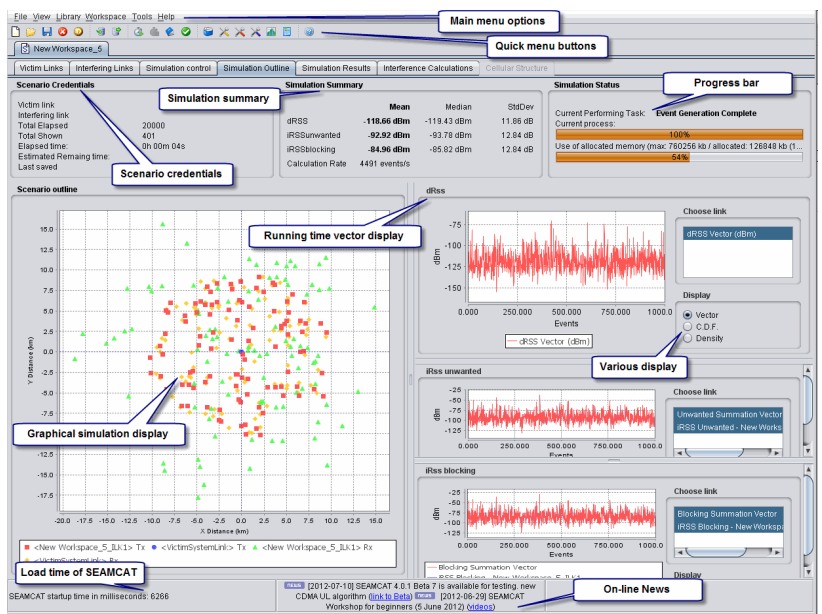

Fig 17: SEAMCAT output window

\section{FUTURE DIRECTIONS}

The spectrum utilization rate increases with DSA and use of licensed spectrum, but it impose treats to security of communication system that implies the requirement of new security protocols and authentication information exchange between Cognitive Radio's. There must be a seamless handover of channel by secondary user when the primary user demands its frequency channel or band. Cognitive Radio should be evaluated and demonstrated in a real world wireless radio environment, to account the different wireless radio environment loses in spectrum sensing and its impact over the Quality of Service and interference at primary user.

\section{CONCLUSION}

Cognitive radio is a futuristic technology that will utilize the spectrum efficiently, and globalize the radio environment for different band users which use different techniques for transmission. The cooperative sensing method is much effective and overcome demerits of non-cooperative sensing method. Cooperative sensing decreases the chances of misdetection and false alarm considerably, cooperation can solve hidden primary user problem and it can decrease sensing time. The high data rate will be achieved by underlay power control techniques while keeping interference low. In spectrum detection techniques, the energy detection based approach is least accurate and complex; and the most accurate and complex approach is matched filter approach. The waveform based approach is as much accurate as matched filter approach but fewer complexes than it. From software tools, SEAMCAT can calculate the interference probability of different communication system with number of propagation models and evaluate different energy detection thresholds and sharing of frequency for Cognitive Radio as secondary user or interferer but it is generic simulation environment for Cognitive Radio and only energy detection can be used. A better analysis could be done with SystemVue, GNU Radio and MATLAB due to compatibility with radio hardware by which analysis could be done in a real environment and with programming, all spectrum sensing techniques for different communication systems can be implemented.

\section{ACKNOWLEDGEMENT}

First author is highly thankful to Dr. Davinder Pal Sharma, DSP Research Lab, University of West Indies; Dr. B.P. Patil, Army Institute of Technology, Pune and Dr. Suyeb Ahmed Khan for providing useful information as well as critical remarks. The valuable help rendered by Hardeep Singh, Research Fellow, DSP Research Lab, Department of Electronic Technology, GNDU Amritsar and Harjit Pal Singh, Research scholar, NIT Jalandhar, India are also acknowledged.

\section{REFERENCES}

[1] Analog Radio invention, online: www.niksun.com.

[2] Cognitive Radio Technology by Bruce A.Fette, 2006, Elsevier Inc.

[3] J. Mitola III, "Software radios: Survey, critical evaluation and future directions," IEEE Aerospace and Electronic Systems Magazine, vol. 8, pp. 25-36, Apr. 1993.

[4] J. Mitola III, "Cognitive radio for flexible mobile multimedia communications," in Proceedings of the IEEE International Workshop on Mobile Multimedia Communications, SanDiego, CA, USA, vol. 1, pp. 3-10, Nov. 1999.

[5] J. Mitola III, "Cognitive radio: An integrated agent architecture for software defined radio." $\mathrm{PhD}$ thesis, Royal Institute of Technology (KTH), Stockholm, Sweden, May 2000.

[6] 5G Based on Cognitive Radio by Cornelia-Ionela Badoi, Neeli Prasad, Victor Croitoru, Ramjee Prasad in Wireless Personal Communication (2011) 57, pp. 441-464.

[7] S. Haykin, "Cognitive radio: Brain-empowered wireless communications," IEEE Journal on Selected Areas in Communications, vol. 23, pp. 201-220, Feb. 2005.

[8] Advances in Cognitive Radio Networks: A Survey by Beibei Wang and K. J. Ray Liu, IEEE Journal Of Selected Topics In Signal Processing, Vol. 5, No. 1, February 2011.

[9] Cognitive radio: An intelligent wireless communication system, MarjaMatinmikko editor, RESEARCH REPORT VTT-R-02219-08.

[10] Cognitive Radio Communications and Networks by Alexander M. Wyglinski, Maziar Nekovee,Y. Thomas Hou.

[11] LO Leakage Based Approach for interference based sensing,www.eecs.berkeley.edu/ dtse/3r_ben_dyspan 05 . pdf [online].

[12] Cognitive Radio, Software Defined Radio, and Adaptive Wireless Systems by HÜSEYIN ARSLAN, University of South Florida, Tampa, FL, USA.

[13] Multitaper Based Spectrum Sensing for Cognitive Radio: Design and Performance by Q. T. Zhang, Department of Electronic Engineering, City University of Hong Kong in IEEE-2011.

[14] Cognitive Radio Networks by Kwang-Cheng Chen and Ramjee Prasad (c) 2009 John Wiley \& Sons Ltd.

[15] S. M. Haykin, Cognitive radio and radio networks. INFWEST seminar in Helsinki, 27-28 June 2007. 
[16] N. Hoven and A. Sahai, "Power scaling for cognitive radio," in Proceedings of WirelessCom 2005, pp. 250255, June 2005.

[17] K.-C. Chen, Y.-J.Peng, N. Prasad, Y.-C. Liang, S. Sun, 'Cognitive Radio Network Architecture: Part I GeneralStructure', ACM ICUMIC, Seoul, 2008.

[18] K.-C. Chen, Y.-J.Peng, N. Prasad, Y.-C. Liang, S. Sun, 'Cognitive Radio Network Architecture: Part II TrustedNetwork Layer Structure', ACM ICUMIC, Seoul, 2008.

[19] Spectrum sharing radios by DanijelaCabric, Ian D. O'Donnell, Mike Shuo-Wei Chen, and Robert W. Brodersen in IEEE CIRCUITS AND SYSTEMS MAGAZINE SECOND QUARTER 2006.

[20] Cognitive Radio Architecture Evolution by Joseph Mitola, III,Proceedings of the IEEE| Vol.97,No.4,April 2009 page 626-641.

[21] C. Chang, J.Wawrzynek, and R.W. Brodersen, "BEE2: A high-end reconfigurable computing system," IEEE Design and Test of Computers, vol. 22, June 2005.

[22] Xilinx, "FPGA and CPLD solutions from Xilinx, Inc." [Online]: www.xilinx.com/.

[23] M. Steyaert, B. D.Muer, P. Leroux, M. Borremans, , and K.Mertens, "Low-voltage low-power CMOS-RF transceiver design," IEEE Transactions on Microwave Theory and Technology, vol. 50, Jan. 2002.

[24] P. Zhang et al., "A single-chip dual-band directconversion IEEE $802.11 \mathrm{a} / \mathrm{b} / \mathrm{g}$ WLAN transceiver in 0.18 micron CMOS," IEEE Journal of Solid-State Circuits, vol. 40, Sept. 2005.

[25] ANSI/IEEE Std 802.11, 1999 Edition, IEEE Std., Jun. 2003.

[26] T. Instruments, TMS320VC5471 Fixed-Point Digital Signal ProcessorData Manual, Dec. 2002.
[27] calradio.calit2.net/MobiEval13-jow_prelim.pdf-CalRadio: A Portable, Flexible 802.11 Wireless Research Platform by Aaron Jow, Curt Schurgers,Douglas Palmer.

[28] KUAR: A Flexible Software-Defined Radio Development Platform, G. J. Minden, J. B. Evans, L. Searl, D. DePardo, V. R. Petty, R. Rajbanshi, T. Newman, Q. Chen, F. Weidling, J. Guffey, D. Datla, B. Barker, M. Peck, B. Cordill, A. M. Wyglinski and A. Agah, Information Technology and Telecommunications Center, The University of Kansas, Lawrence.

[29] KUAR Control Processor, online: www.research. microsoft.com/enus/um/redmond/events/.../evans.ppt.

[30] Building A Cognitive Radio Network Testbed by Zhe Chen, Nan Guo, and Robert C. Qiu in IEEE 2011.

[31] Small Form Factor (SFF) Software-Defined Radio (SDR) Development Platform,online: www.dsp-fpga.com/rsc

[32] Universal software radio peripheral (USRP) and USRP2 provided by Ettus Research, online: www.ettus.com

[33] Design Of Warp: A Wireless Open-Access Research Platform, Patrick Murphy, Ashu Sabharwal and Behnaam Aazhang, Rice University, Department of Electrical and Computer Engineering, 6100 Main St., Houston.

[34] A. P. Hulbert "Spectrum sharing through beacons", in Proceedings of PIMRC 2005, pp. 989-993, September 2005.

[35] SystemVue's developed and tested CR whitespace algorithm, www.agilent.com/find/eesof-cognitivewhitepaper.

[36] GNU Radio Companion, online: www.gnu.org .

[37] Spectrum Engineering Advanced Monte Carlo Analysis Tool, online: http://www.ero.dk/seamcat 